$$
\text { CONF-970503--276 }
$$

\title{
A Coupled-Bunch Ion Instability in the SLC Electron Damping Ring *
}

\author{
P. Krejcik \\ Stanford Linear Accelerator Center, Stanford University, Stanford CA 94309
}

\section{DISCLAIMER}

\begin{abstract}
This report was prepared as an account of work sponsored by an agency of the United States Government. Neither the United States Government nor any agency thereof, nor any of their employees, makes any warranty, express or implied, or assumes any legal liability or responsibility for the accuracy, completeness, or usefulness of any information, apparatus, product, or process disclosed, or represents that its use would not infringe privately owned rights. Reference herein to any specific commercial product, process, or service by trade name, trademark, manufacturer, or otherwise does not necessarily constitute or imply its endorsement, recommendation, or favoring by the United States Government or any agency thereof. The views and opinions of authors expressed herein do not necessarily state or reflect those of the United States Government or any agency thereof.
\end{abstract}

\begin{abstract}
Under abnormally poor vacuum conditions in the electron damping ring, described in a companion paper at this conference, an emittance blowup was observed related to a transverse instability. The instability was only observed with two bunches in the ring, and could not be seen with single bunch operation, even at the same total beam current as for the two bunches. Measurements of the transverse Beam Transfer Function indicate strong coupling between the two bunches when ions are present. This is described in terms of a simple model where the oscillating ion cloud provides the coupling between the two bunches. The frequency shift due to coupling can also cause the ion cloud to become unstable, and disappear, when the betatron tune of the electron bunch is just below the $1 / 2$ integer.
\end{abstract}

Contributed to the 1997 Particle Accelerator Conference, Vancouver, B.C., Canada, May 12-16, 1997

* Work supported by Department of Energy contract DE-AC03-76SF00515.

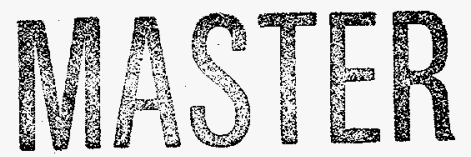




\section{A Coupled-Bunch Ion Instability in the SLC Electron Damping Ring ${ }^{*}$ P. Krejcik, Stanford Linear Accelerator Center, Stanford University, Stanford, CA 94309 USA}

\section{Abstract}

Under abnormally poor vacuum conditions in the electron damping ring, described in a companion paper at this conference, an emittance blowup was observed related to a transverse instability. The instability was only observed with two bunches in the ring, and could not be seen with single bunch operation, even at the same total beam current as for the two bunches. Measurements of the transverse Beam Transfer Function indicate strong coupling between the two bunches when ions are present. This is described in terms of a simple model where the oscillating ion cloud provides the coupling between the two bunches. The frequency shift due to coupling can also cause the ion cloud to become unstable, and disappear, when the betatron tune of the electron bunch is just below the $1 / 2$ integer.

\section{INTRODUCTION}

An instability has been observed in the $1.2 \mathrm{GeV}$ SLC electron damping ring relating to the poor vacuum conditions following an accidental venting and contamination of the ring. The instability causes a large amount of emittance blowup, particularly in the vertical plane, which has been addressed in a companion paper at this conference[1]. Many of the observations point to a multi-bunch effect rather than a single bunch emittance growth phenomenon. The signature of the instability, the appearance of strong vertical betatron sidebands in the bunch spectrum, persists down to low intensities of $1 \cdot 10^{10}$ total number of electrons when two bunches are present. However, single bunch intensities as high as $4 \cdot 10^{10}$ electrons do not show such sidebands in the bunch spectrum. The emittance growth of single bunches also clearly decreases at lower intensities, whereas the emittance growth with two bunches is large over a wide range of operating currents.

One important conclusion from the observations of the ion effects in the SLC damping ring is that the ions are only able to be captured during a brief interval after injection while the beam is large. Once the beam has damped to small dimensions the critical mass for an ion to be captured gets quite large. Since we continue to observe ion related emittance growth at long store times this implies that the ion cloud that formed after injection has also shrunk in transverse dimension with the beam. If the ion cloud had not shrunk with the beam those ions would have large amplitude oscillations and be lost from the beam.

This paper concentrates on the coupling aspect of the instability. Measurement techniques were developed to distinguish the coupling from other beam

\footnotetext{
- Work supported by Department of Energy Contract DE-AC03-76SF00515.
}

instability modes. A simple model is used to demonstrates how the measured transverse beam transfer function can distinguish between coupled and non-coupled bunches. The change in the instability behavior with the vertical tune can also be interpreted in light of the coupling mechanism. The tune shift of the beam and the shift in frequency of the coherent dipole oscillations of the ion cloud depend upon the modes that are possible in the presence of coupling. Under certain tune conditions the ion cloud is found to be able to shake free of the beam and the instability stops.

\section{MEASUREMENT METHODS}

The amplitude response of the beam gives some indications of the presence of ions, as described in reference[1]. At CESR the technique has been developed to infer the ion density from the amplitude response using a linear model [2]. A complete characterization of the Beam Transfer Function (BTF) in the presence of ions requires the measurement of both phase and amplitude response, as has been performed for unbunched beams in the CERN antiproton rings [3]. The output from the network analyzer drives a broadband transverse kicker through a $50 \mathrm{~W}$ amplifier, as illustrated in figure 1. A stripline kicker is used so the current measured in the stripline is proportional to the kick received by the beam and is used as the reference signal for the BTF. The beam response is measured at another stripline and low-pass filtered to remove the power generated at high frequencies by the short bunches. The signal to noise ratio is improved by using a low noise pre-amplifier in the signal path.

The network analyzer used on these measurements is a conventional swept frequency instrument that acquires the data over several seconds. The beam is stored during this time and has therefore reached its equilibrium emittance. Although data can be retrieved while the damping ring is cycling through many injection and extraction cycles, it is difficult to interpret because of the varying beam conditions. The data presented here is all for stored beams. Plans are under way to use a synthesized sweep instrument that can be gated on during specific intervals of the store.

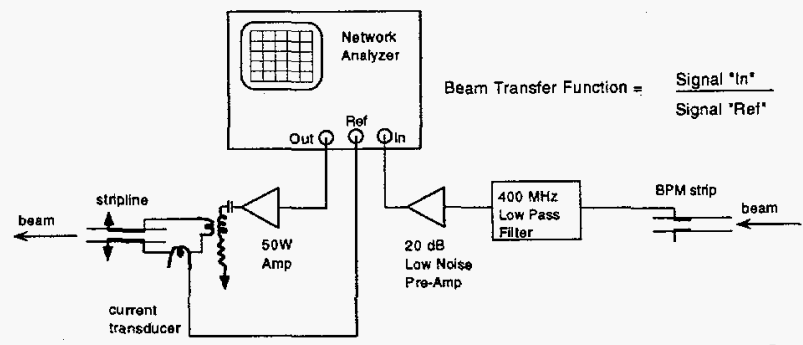

Figure 1: Measurement of the transverse beam transfer function with a network analyzer driving the beam and measuring the response at a stripline monitor. 


\section{DISCLAMMIER}

Portions of this document may be illegible in electronic image products. Images are produced from the best available original document. 


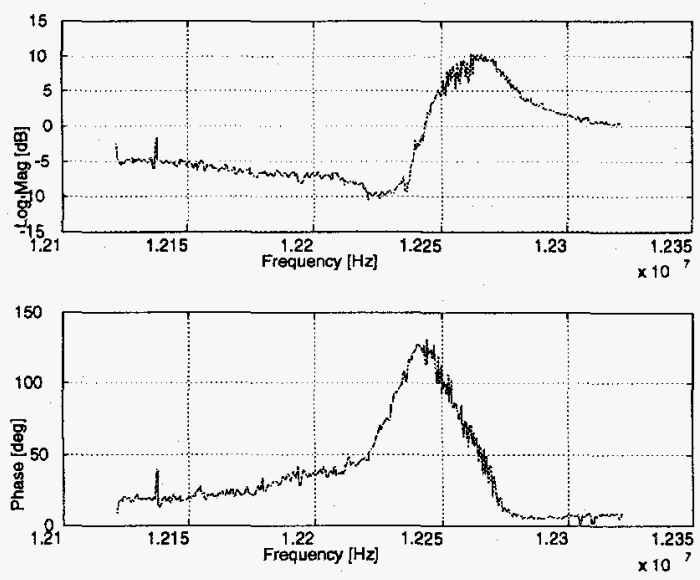

Figure 2: Measured magnitude and phase response for two bunches of $4 \cdot 10^{10}$ electrons each at $f_{0}+v_{\mathrm{v}}$.

\section{MEASUREMENT RESULTS}

The first case shown in figure 2 is for two bunches of $4 \cdot 10^{10}$ electrons each, which is close to the nominal intensity for SLC operation. The amplitude and phase response do not show a single resonance response as might be expected. The phase does not make a net change as it passes through the resonance, but returns close to its starting value. The tune spread in the electron beam can be deduced from the width of the amplitude response.

The second case in figure 3 is for a single bunch with the same bunch charge as in the previous example. The tune spread in the amplitude response is comparable in both cases. However, a large difference is seen in the phase response between the single and two bunch cases. The phase makes a net change as the frequency is swept through the resonance in the single bunch case.

The third case in figure 4 is again for a single bunch but at a reduced intensity of $1 \cdot 10^{10}$ electrons. The tune spread seen in the amplitude response is reduced, but the phase response shows the same behavior as for high intensities, with a net phase change across the resonance.

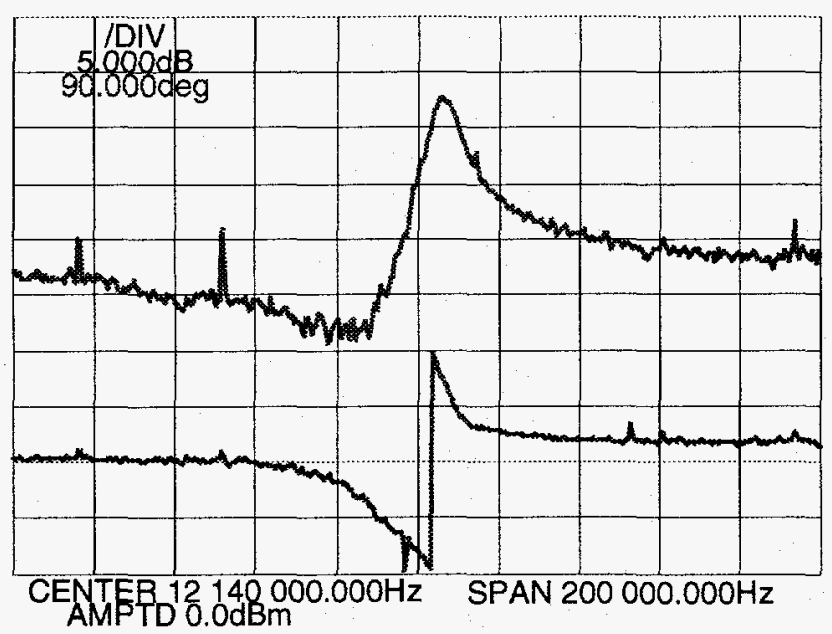

Figure 3: Measured magnitude and phase response for one bunch of $4 \cdot 10^{10}$ electrons at $f_{0}+v_{v}$.

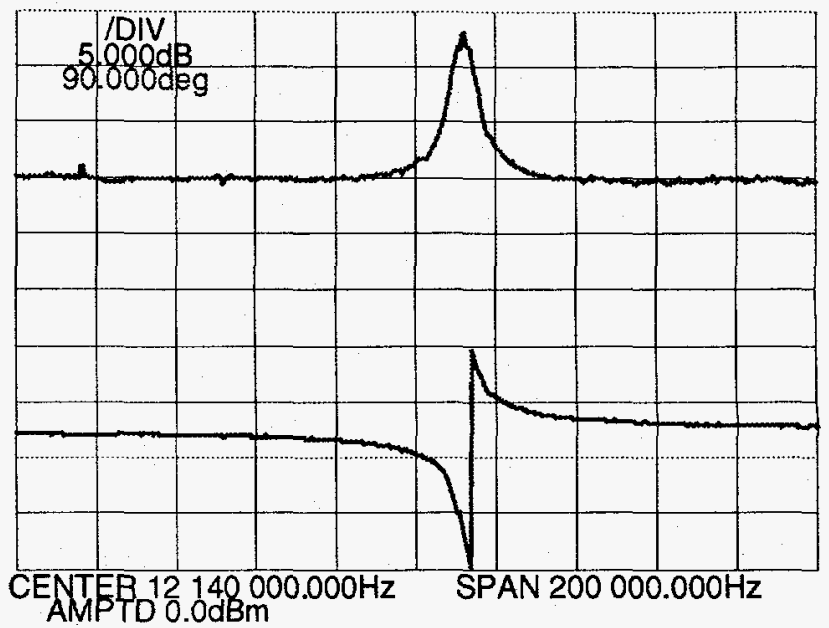

Figure 4: Measured magnitude and phase response for one bunch of $1 \cdot 10^{10}$ electrons at $\mathrm{f}_{0}+v_{\mathrm{v}}$.

\section{COUPLED BUNCH MODEL}

In a simple model the transverse dipole motion of the bunch can be described as an oscillator with natural frequency $\omega_{n}$ and damping ratio $\zeta$. If coupling is included between the two bunches the coupling force is represented by the coupling frequency $\omega_{c}$ and for completeness a damping ratio $\zeta_{c}$ for the coupling is also included. The equations of motion for the phases of bunch $1, \phi_{1}$, and bunch $2, \phi_{2}$, are

$$
\begin{aligned}
& \ddot{\phi}_{1}+\left(\zeta+\zeta_{c}\right) \dot{\phi}_{1}+\left(\omega_{n}^{2}+\omega_{c}^{2}\right) \phi_{1}+\omega_{c}^{2} \phi_{2}+\zeta_{c} \dot{\phi}_{2}=0 \\
& \ddot{\phi}_{2}+\left(\zeta+\zeta_{c}\right) \dot{\phi}_{2}+\left(\omega_{n}^{2}+\omega_{c}^{2}\right) \phi_{2}+\omega_{c}^{2} \phi_{1}+\zeta_{c} \dot{\phi}_{1}=0
\end{aligned}
$$

The coupled bunch motion has two modes, an 0 -mode $(n=0)$ and a $\pi$-mode $(n=1)$ where the two bunches are in phase or out of phase respectively. The frequency of these two modes is given by

$$
\omega^{2}=\omega_{n}^{2} \pm \omega_{c}^{2}
$$

where the $\pi$-mode is shifted up in frequency due to coupling. Note that this amounts to an additional tune shift above that already caused by the focusing effect of the ions on a single bunch.

Equation (1) represents a fourth-order system from which the transfer function can be deduced. The amplitude and phase response versus frequency (Bode plot) is shown in figure 5 for this system. The frequency scale is normalized so that the natural frequency $\omega_{n}=1$ in the plot. The characteristic behavior of the coupled system is that the frequency sweeps through two resonances, with the phase increasing at the first resonance and then decreasing to the starting value as the second resonance is passed.

The modeled behavior of the fourth-order system resembles very closely the measured response of the two bunch system in figure 2 . Note also that the measured two bunch response was at a sideband of an odd revolution harmonic, corresponding to a $n=1$ mode for the bunch oscillation.

As a comparison, a similar frequency analysis is shown for the single bunch case. The simple oscillator for a single bunch is a second-order system whose transfer function may be simply written using control system formalism as 

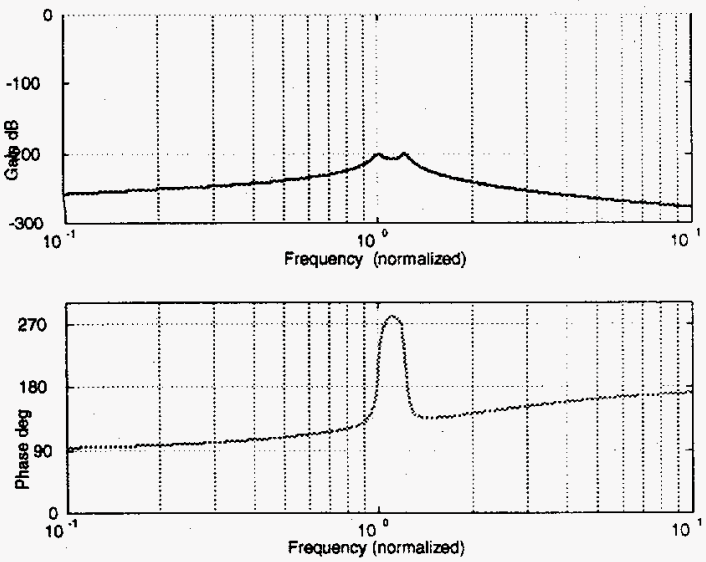

Figure 5: The predicted magnitude and phase response for a 4th-order coupled oscillator model.

$$
G(s)=\frac{\omega_{n}^{2}}{s^{2}+2 \zeta \omega_{n} s+\omega_{n}^{2}}, \quad s=j \omega
$$

The transfer function has the well-known frequency response of a second-order system, shown in figure 6. Comparing with the measured single bunch response in figures 3 and 4 one can see a similar net phase change across the resonance.

The control system formalism is a useful tool for analyzing the coupling since it is evident that the coupled oscillators can be modeled as two sequential transformations of the type in equation (3). The first transformation corresponds to the single bunch response and the second to the coupling term, so that the complete transfer function can be written as

$$
G(s) \times G_{c}(s)=\frac{\omega_{n}^{2}}{s^{2}+2 \zeta \omega_{n} s+\omega_{n}^{2}} \times \frac{\omega_{c}^{2}}{s^{2}+2 \zeta_{c} \omega_{c} s+\omega_{c}^{2}}
$$

which represents the 4th-order system in equation (1).

\section{ION CLOUD BEHAVIOR}

The modeling above supports the existence of coupling between the bunches. It is further concluded that the coupling is due to ions, since the effect was not seen before the vacuum contamination occurred and the coupling is also not seen in the SLC positron damping ring where no ion trapping occurs. In the introduction it was noted that the ion cloud must shrink with the beam as it damps, in order to account for the persistence of the ions into the store. The small $(\sim 70 \mu \mathrm{m})$ beam sizes in the damping ring are good candidates for two-stream instabilities to occur as described in plasma physics. That is, the centroids of the electron bunch and the ion cloud will begin to oscillate with respect to each other. The oscillations are self limiting since at larger amplitudes the electron bunch samples the nonlinear $1 / \mathrm{r}$ field of the ion cloud. The classical multi-turn instability is discussed, for example in references $[4,5]$.

If we try and include the ion cloud as a third bunch in the model we end up with a sixth-order system describing the phases $\phi_{1}, \phi_{2}$ and $\phi_{3}$. This is of limited use since the measurement system only detects the position of the electron bunches and not the ion
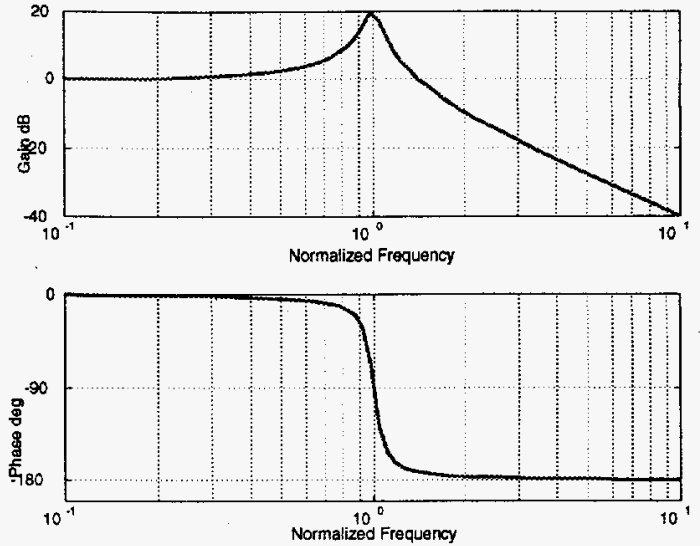

Figure 6: The predicted magnitude and phase response for a 2 nd-order single oscillator model.

cloud. However, it is useful to note that there are three oscillation modes present, $n=0,1$ and 2 . In the $n=0$ mode the three bunches are in phase, but in the $n=1$ and $n=2$ modes the two electron bunches are out of phase and the ion cloud is in phase with either one bunch or the other. Since the ion cloud is always out of phase with one bunch its frequency is always shifted upward according to equation (2).

The expected frequency shift of the ions can account for some of the tune behavior seen with the beam. It has been observed that operating with the vertical tune just below the $1 / 2$ integer resonance cures some of the emittance growth problems due to the instability. If the ion cloud dipole mode frequency is shifted upward by the coupling the ions will be sitting in closer proximity to the $1 / 2$ integer resonance and their amplitude may grow until they are expelled from the beam. The sudden tune jumps observed under these conditions might also be due to changes in the dipole modes as the ion density changes.

Only the coherent dipole motion of the electron bunches have been considered so far. Transverse quadrupoles can also be driven by the coupling in the ion space charge, but can remain hidden from normal dipole pickups, as was the case with the antiproton beams in references $[3,5]$.

\section{REFERENCES}

1. P. Krejcik, D. Pritzkau, T. Raubenheimer, M. Ross, F. Zimmermann, "Ion Effects in the SLC Electron Damping Ring", Paper 2V18, these proceedings.

2. D. Sagan, A. Temnykh, "Observations of the Coherent Beam-Ion Interaction in the CESR Storage Ring", NIM. A344, p.459-469, (1994).

3. G. Carron et al., "Observation of Transverse Quadrupole Mode Instabilities in Intense Cooled Antiproton Beams", Proc. 1989 PAC, IEEE p.803, (1989).

4. G. Koshkarev and P. Zenkevich, "Resonance of Coupled Transverse Oscillations in Two Circular Beams", Particle Accelerators 3, p. 1, (1972).

5. R. Alves Pires et al., "On the Theory of Coherent Instabilities due to Coupling between a Dense Cooled Beam and Charged Particles from the Residual Gas", Proc. 1989 PAC, IEEE p.800, (1989). 